\title{
MINIMIZATION APPROACH TO LIMIT SOLUTIONS OF PLATES
}

\author{
Wei H. YANG \\ The University of Michigan, Ann Arbor, MI 48109, USA \\ Received 24 October 1980 \\ Revised manuscript received 8 January 1981
}

\begin{abstract}
Limit analysis which predicts the ultimate load carrying capacity of a structure or machinery provides very useful information especially for designs that must survive accidents and abnormal conditions. In this paper, maximization of lower bound formulation and minimization of upper bound formulation are presented as primal and dual problems respectively. The dual problem is solved by a minimization procedure for circular, triangular and square plates with three types of boundary conditions and two types of loadings. A detailed parametric analysis for a cracked plate is also given to demonstrate the effectiveness of this approach.
\end{abstract}

\section{Introduction}

Plastic design of structures $[1,12]$ has generally been considered superior to the conventional elastic design. It gives a more uniform strength distribution and therefore reduces the weight of the structures. It is less sensitive to imperfections and misalignments of structural components during construction. It provides more realistic prediction of structural behavior under severe and unexpected conditions such as earthquakes and accidents.

Analysis of plastic design is more difficult. Even with the powerful existing elastoplastic computer programs [2,3], plastic analysis of large and complex structures remains a formidable task. Costs of analytic man power and computer time often offset the gain in material savings for ordinary structures. This is the reason why most of the construction codes are still based on elastic analysis.

With light-weight design now made necessary for vehicular structures, the plastic range of the materials should be considered in designs. With higher demand on reliability of earthquake proof structures and accident safe nuclear installations, more thorough elastic-plastic analysis and prediction of ultimate strength must be understood.

Limit analysis has a simple mathematical structure yet describes a realistic nonlinear behavior. When an exact solution is obtainable by the analysis, it gives the ultimate load limit of a structure. Approximate solutions provide bounds on the limit loads.

For convenience of presenting the ideas in this paper, the classical definitions of statical and kinematical admissibilities [4] are slightly modified and augmented with the additional constitutive admissibility. A solution of any mechanics problem satisfies simultaneously the equilibrium equation, the kinematical compatibility condition and a mathematical description of material behavior (constitutive relation). Consider a function space of mechanics solutions. The solutions satisfying the equilibrium equation and its boundary conditions, if present, 
belong to a point set $S$ in the space, named statically admissible. The solutions derived from a single-valued displacement field and its boundary conditions form another set $K$ named kinematically admissible. Solutions which do not violate the constitutive equation (or relation) establish the constitutively admissible set $C$. The exact solution, or solutions if not unique, lies in the intersection of these three sets.

Limit analysis studies the solutions in the intersections of two of the three sets. The lower bound solutions lie in $S \cap C$. The upper bound solutions lie in either $K \cap C$ or $K \cap S$. These statements agree with the well known lower and upper bound theorems [5] although they may not be in the same wording. A limit solution is an extreme point of one of these convex sets defined by either the lower or the upper bound solutions.

The plates under consideration are assumed to be stiff and have well defined limit values of bending moment. The modern structural plates such as the sandwich plates [6] and composite plates [7] are designed to have these properties. High stiffness implies infinitesimal elastic deformation and enables the equilibrium equation to be written in the undeformed coordinates and therefore to be linear. A yield criterion without hardening models a class of plate behavior in which infinite curvature can occur under a constant yield moment. An initially smooth flat plate may develop a displacement field containing ridge lines which allow discontinuous gradients. These ridge lines deform like piano hinges.

Limit analysis predicts the initial collapse condition under which the plate deforms continuously with non increasing load. The initial collapse may soon stabilize when large deformation of the plate causes part of the load to be carried by the membrane stresses. The large deformation is outside the scope of limit analysis.

The formulation starts with the lower bound theorem from the set point of view. Although the resulting maximization problem is difficult to solve, it gives a clear concept of uniqueness of optimality and possible nonuniqueness of optimal solutions. An upper bound to the optimality is obtained by the virtual work principle and by forcing the constitutive inequality to the bounds everywhere. This leads to a minimization problem.

Several simple solutions for circular, triangular and square plates with various boundary conditions and loads and a detailed analysis for a cracked plate with various crack angles and lengths are obtained by minimization.

\section{Lower bound formulation}

The equilibrium equation for plates with a frequently used sign convention is given in [8]

$$
\frac{\partial^{2} M_{x x}}{\partial x^{2}}-2 \frac{\partial^{2} M_{x y}}{\partial x \partial y}+\frac{\partial^{2} M_{y y}}{\partial y^{2}}=-q(x, y) \text { in } D,
$$

where $M_{x x}, M_{x y}, M_{y y}$ are moment components and $q(x, y)$ is the load distribution function in the domain $D$.

Let $(\partial / \partial x, \partial / \partial y)$ be a two dimensional vector operator and let the moment components be arranged in a symmetric $2 \times 2$ matrix denoted by $M$. The above equation can be written in the compact form, 


$$
\nabla \cdot(\nabla \cdot M)=q \quad \text { in } D, \quad M=\left[\begin{array}{rr}
-M_{x x} & M_{x y} \\
M_{x y} & -M_{y y}
\end{array}\right]
$$

where $\cdot$ is the inner product of vectors or a vector and a matrix.

Eq. (2) defines a set in the space $\mathrm{R}^{2 \times 2}$. The set is convex since for any two points $M_{1}$ and $M_{2}$ in the set, $\alpha M_{1}+(1-\alpha) M_{2}(0 \leq \alpha \leq 1)$ is also in the set, a consequence of the linear operator.

Consider a yield criterion that models the limiting behavior of the plastic plate by the inequality,

$$
f(M) \leq 0,
$$

where $f$ is a map from $\mathbf{R}^{2 \times 2}$ to $\mathbb{R}$. We need not specify $f$ now except to require that $f$ is convex. Therefore, the relation (3) also defines a convex set in $\mathbb{R}^{2 \times 2}$. The points $M$ lying in the intersection of the sets defined by (2) and (3) are called lower bound solutions. Lower bound solutions form a convex set since the intersection of convex sets is convex.

If $q$ is given in the form of proportional loading such that $q=q_{0} \phi(x, y)$ where $q_{0}>0$ and $\phi(x, y)$ is a given integrable function, the constrained maximization problem,

$$
\begin{array}{ll}
\max & q_{0} \\
\text { s.t. } & \nabla \cdot(\nabla \cdot M)=q_{0} \phi, \quad f(M) \leq 0
\end{array}
$$

has a solution $\left(M, q_{0}\right) \in \mathbb{R}^{2 \times 2} \times \mathbb{R}$. The boundary conditions on moment and shear if present should also be included in the constraint set. Some problems may not have these static boundary conditions. $q_{0}$ can be uniquely determined, but $M$ may not be unique. These are the properties of the convex programming problem given in (4). A proportional loading is also called a one parameter distribution.

If $q$ has a finite $N$-parameter distribution such that $q=\Sigma_{i=1}^{N} q_{i} \phi_{i}(x, y), q_{i}>0$, we have another maximization problem,

$$
\begin{array}{ll}
\max & \iint_{D} q \mathrm{~d} A \\
\text { s.t. } & \nabla \cdot(\nabla \cdot M)=q, \quad f(M) \leq 0,
\end{array}
$$

which has a unique maximum for the integral $\iint_{D} q \mathrm{~d} A$.

The problems (4) and (5) are in principle solvable by a finite dimensional space approximation but the numerical tasks are formidable. Nevertheless, we know the solution exists and the optimality is unique for each problem. These assertions provide the basis for the method of solution to the upper bound formulation derived in the following section from (4) by the virtual work principle.

\section{Upper bound formulation}

We shall now satisfy the equilibrium equation (2) in a weak sense (virtual work) such that 


$$
\iint_{D} w \nabla \cdot(\nabla \cdot M) \mathrm{d} A=\iint_{D} w q \mathrm{~d} A \text { for all } w \in W
$$

where $W$ is the space of real, kinematically admissible functions which are continuous in $D$ and satisfy certain boundary conditions.

The left-hand side of (6) can be manipulated into another form by the use of Green's theorem

$$
\begin{aligned}
\iint_{D} w \nabla \cdot(\nabla \cdot M) \mathrm{d} A= & \iint_{D} \nabla \cdot(w \nabla \cdot M) \mathrm{d} A-\iint_{D}(\nabla \cdot M) \cdot \nabla w \mathrm{~d} A \\
= & \oint_{\partial D} w n \cdot(\nabla \cdot M) \mathrm{d} S-\iint_{D} \nabla \cdot(M \cdot \nabla w) \mathrm{d} A \\
& +\iint_{D} M: \nabla \nabla w \mathrm{~d} A \\
= & \oint_{\partial D}[w n \cdot(\nabla \cdot M)-n \cdot M \cdot \nabla w] \mathrm{d} S+\iint_{D} M: \nabla \nabla w \mathrm{~d} A
\end{aligned}
$$

where $n$ is the unit normal vector along the boundary $\partial D$ and : denotes the inner product of two matrices such that

$$
M: \nabla \nabla w=-M_{x x} \frac{\partial^{2} w}{\partial x^{2}}+2 M_{x y} \frac{\partial^{2} w}{\partial x \partial y}-M_{y y} \frac{\partial^{2} w}{\partial y^{2}} .
$$

The line integral in (7) vanishes for all types of natural boundary conditions [10]. These include the simply supported, the clamped and the free edge conditions as well as the combinations of these boundary conditions.

Consider the case where $q$ has a one parameter distribution such that $q=q_{0} \varphi(x, y)$. We can write (6) in the form

$$
q_{0}(M)=\iint_{D} M: \nabla \nabla w \mathrm{~d} A / \iint_{D} \varphi w \mathrm{~d} A
$$

provided that $\iint_{D} \varphi w \mathrm{~d} A \neq 0$. There is no loss of generality if we set $\iint_{D} \varphi w \mathrm{~d} A>0$. The problem (4) becomes

$$
\begin{array}{ll}
\max & q_{0}(M), \\
\text { s.t. } & f(M) \leq 0 .
\end{array}
$$

The solution $M$ which maximizes $q_{0}(M)$ gives the limit load $q_{0 L}$. This maximization problem is by no means easy to solve and there exists no satisfactory algorithm for its solution. We shall construct the dual of (10) which leads to a minimization problem. Approximate solutions of the minimization problem give upper bounds to the limit load $q_{0 L}$.

Note that $M$ in (10) is constitutively admissible therefore it lies inside or on the yield 
surface. Consider a state $M^{*}$ which is on the yield surface such that $f\left(M^{*}\right)=0$ and is associated with a $w$ leading to the yield state. The inequality

$$
\left(M^{*}-M\right): \nabla \nabla w \geq 0
$$

states the well-known condition of Drucker [15] on stable materials. Using (11), we have an upper bound to $q_{0}(M)$ such that

$$
q_{0}(M) \leq \iint_{D} M^{*}: \nabla \nabla w \mathrm{~d} A / \iint_{D} \varphi w \mathrm{~d} A=q^{*}(w),
$$

where the form of $M^{*}(w)$ in $q^{*}(w)$ depends on the specific $f(M)$. The least upper bound $q_{L}^{*}$ can be obtained by minimizing $q^{*}(w)$ over all kinematically admissible $w(x, y)$ such that

$$
q_{L}^{*}=\min _{w} \iint_{D} M^{*}: \nabla \nabla w \mathrm{~d} A / \iint_{D} \varphi w \mathrm{~d} A
$$

Mathematically, (13) is called the dual problem of (10) which is called the primal problem. For some $f(M), M^{*}$ can be made explicit and the dual problem involves only the kinematical variable $w(x, y)$ which is easier to visualize than $M(x, y)$. For simple domains, $w(x, y)$ can be parameterized and the minimization can be carried out in a small parameter space. For complex domains, the correct $w$ may be iteratively approached by a sequence of trial functions in a finite dimensional space. Even if $w$ is not optimal, $q_{L}^{*}$ so obtained by (13) always bounds the exact limit load from above such that

$$
q_{L}^{*} \geq q_{0 L} .
$$

The examples in the following sections belong to the class of simple domains. A specific yield function [11]

$$
f(M)=\|M\|_{2}^{2}-M_{n}^{2}=\lambda_{\max }^{2}(M)-M_{0}^{2}
$$

is used where $\lambda_{\max }^{2}(M)$ is the largest eigenvalue of $M^{2}$ and $M_{0}$ is the constant yield moment of the plate. The function is convex and $\lambda_{\max }$ occurs in the major principal direction of $M$. It is physically reasonable to assume that along the lines of maximum moment ridges will form.

For a given $\phi$, we may choose a $w(x, y)$ in the following way. Let the domain $D$ be divided into finite number of subdomains $D_{i}$ such that

$$
D=\bigcup_{i=1}^{N} D_{i} \text {. }
$$

Within each $D_{i}$, there exists a local curvilinear orthogonal coordinate system $(\xi, \eta)$ such that $M_{\xi \eta}^{*}=0$. If $w$ is linear along one coordinate say $\xi$, this direction corresponds to the minor principal direction along which the moment is less than $M_{0}$. Along the major principal direction $\eta,\left|M_{\eta \eta}^{*}\right|=M_{0}$ and $\partial^{2} w / \partial \eta^{2} \neq 0$. If $w$ is linear in both directions, the subdomain deforms like a rigid body. 
The function $w(x, y)$ must be continuous and satisfies the kinematical boundary conditions given in (7). The intersections of all $D_{i}(i=1,2, \ldots, N)$ are the ridge lines. Let $(n, s)$ denote the coordinates along each ridge line with $s$ being the line. Then $\partial w / \partial n$ has a discontinuity across the ridge line and $\left|\boldsymbol{M}_{n n}^{*}\right|=\boldsymbol{M}_{0}$.

Since $M^{*}: \nabla \nabla w$ is invariant to coordinate rotation, we may write

$$
M^{*}: \nabla \nabla w=-M_{\xi \xi}^{*} \frac{\partial^{2} w}{\partial \xi^{2}}-M_{\eta \eta}^{*} \frac{\partial^{2} w}{\partial \eta^{2}}
$$

For each choice of $w$, the quotient in equation (13) is an upper bound. We choose $M_{\xi \zeta}^{*}$ and $M_{\eta \eta}^{*}$ to be both on the bounds $\left( \pm M_{0}\right)$ to give

$$
\iint_{D} M^{*}: \nabla \nabla w \mathrm{~d} A \leq M_{0} \iint_{\Omega}\left(\left|\frac{\partial^{2} w}{\partial \xi^{2}}\right|+\left|\frac{\partial^{2} w}{\partial \eta^{2}}\right|\right)|J| \mathrm{d} \xi \mathrm{d} \eta+M_{0} \int_{S}\left|\left[\frac{\partial w}{\partial n}\right]\right| \mathrm{d} s,
$$

where $\Omega$ is the union of all subdomains, where $\nabla \nabla w \neq 0, S$ is the union of all ridge lines and $[\partial w / \partial n]$ is the jump of $\partial w / \partial n . J$ is the Jacobian of the transformation from $(x, y)$ to $(\xi, \eta)$.

We have established an upper bound to $q_{0 L}$ for a kinematically admissible function $w$ such that

$$
q_{0 L} \leq M_{0}\left[\iint_{\Omega}\left(\left|\frac{\partial^{2} w}{\partial \xi^{2}}\right|+\left|\frac{\partial^{2} w}{\partial \eta^{2}}\right|\right)|J| \mathrm{d} \xi \mathrm{d} \eta+\int_{S}\left|\left[\frac{\partial w}{\partial n}\right]\right| \mathrm{d} s\right] / \int_{D} \phi w \mathrm{~d} A=q^{*}(w)
$$

The least upper bound $q_{L}^{*}$ may be obtained by mi.imizing the function $q^{*}(w)$ such that

$$
q_{L}^{*}=\min _{w} M_{0}\left[\iint_{\Omega}\left(\left|\frac{\partial^{2} w}{\partial \xi^{2}}\right|+\left|\frac{\partial^{2} w}{\partial \eta^{2}}\right|\right)|J| \mathrm{d} \xi \mathrm{d} \eta+\int_{S}\left|\left[\frac{\partial w}{\partial n}\right]\right| \mathrm{d} s\right] / \iint_{D} \phi w \mathrm{~d} A .
$$

Eq. (20) is a special case of (13) and is the one used in the examples.

\section{Simple examples}

In order to present a large number of solutions in the limited pages allowed, the detailed derivation for each problem is omitted. They are interesting exercises of interaction between intuition and mathematical optimization process. The choices of functions $w(x, y)$ are guided by intuition to establish a few variable parameters. The computation then proceeds to obtain the values of the parameters that minimize $q^{*}$. The choices of $w(x, y)$ in $\Omega$ are linear and conical which intersect at linear and circular ridge lines. The parameters in $w(x, y)$ which minimize $q^{*}$ characterizes the configurations of collapse mode.

Another interpretation of the above algorithm is the finite element concept. The special finite element method employed here does not fix its mesh system. But the basis function in each element is predetermined. The mesh system is parameterized so that $q^{*}$ is minimized in the parameter space. 
Three shapes of plates, circle, equilateral triangle and square, are included. Each plate is imposed with three cases of boundary conditions: resting, simply supported and clamped edges. The resting edge condition restricts downward displacement but allows the plate to leave the support at places when compressive reaction from the support is absent. In each case, two distribution functions are chosen: $\phi_{1}(x, y)=1$ and $\phi_{2}(x, y)=\delta(x, y)$ where $\delta(x, y)$ is the delta function with origin at the center of each plate. The optimal $q_{L}^{*}$ corresponds to $\phi_{1}$ is the uniform collapse load intensity. The optimal $q_{L}^{*}$ corresponds to $\phi_{2}$ is the magnitude of the concentrated collapse load denoted by $P$.

The integrals in (20) can be easily calculated in the conical subdomains and along the ridge lines since $\partial^{2} w / \partial \eta^{2}$ and $[\partial w / \partial n]$ have simple expressions and can be integrated exactly. There is no contribution by the integration in the remaining domain since $\nabla \nabla w=0$.

The results for all 18 combinations of plate configurations, boundary conditions and load distributions are given in table 1 and table 2 . The meanings of the angles $\theta_{1}$ and $\theta_{2}$ and lengths

Table 1

Uniformly distributed collapse load intensity

\begin{tabular}{lccccc}
\hline $\begin{array}{l}\text { Boundary } \\
\text { condition }\end{array}$ & Shape & $q \ell^{2} / 6 M_{0}$ & $\theta_{1}\left(^{\circ}\right)$ & $\theta_{2}\left(^{\circ}\right)$ & $d / l$ \\
\hline \multirow{4}{*}{ Resting } & Sq & 0.92495064 & 35.78 & 18.44 & 0.72069224 \\
& $\mathrm{Cr}$ & 1.00000000 & - & - & - \\
& $\mathrm{Tr}$ & 2.61836929 & 50.45 & 19.09 & 0.69913792 \\
Simply & $\mathrm{Sq}$ & 1.00000000 & 45.00 & 0.00 & 1.00000000 \\
supported & $\mathrm{Cr}$ & 1.00000000 & - & - & - \\
& $\mathrm{Tr}$ & 2.83179668 & 53.46 & 13.08 & 0.77910598 \\
\multirow{4}{*}{ Clamped } & $\mathrm{Sq}$ & 1.81098918 & 31.05 & 27.90 & 0.60204897 \\
& $\mathrm{Cr}$ & 2.00000000 & - & - & - \\
& $\mathrm{Tr}$ & 4.95895700 & 42.75 & 35.50 & 0.53369682 \\
\hline
\end{tabular}

Table 2

Concentrated collapse load at plate center

\begin{tabular}{lccccc}
\hline $\begin{array}{l}\text { Boundary } \\
\text { condition }\end{array}$ & Shape & $P / M_{0}$ & $\theta_{1}\left({ }^{\circ}\right)$ & $\theta_{2}\left({ }^{\circ}\right)$ & $d / l$ \\
\hline \multirow{4}{*}{ Resting } & $\mathrm{Sq}$ & 6.62741700 & 22.50 & 45.00 & 0.41421357 \\
& $\mathrm{Cr}$ & 6.28318531 & - & - & - \\
& $\mathrm{Tr}$ & 6.92820323 & 30.00 & 60.00 & 0.33333333 \\
Simply & $\mathrm{Sq}$ & 8.00000000 & 45.00 & 0.00 & 1.00000000 \\
supported & $\mathrm{Cr}$ & 6.28318531 & - & - & - \\
& $\mathrm{Tr}$ & 9.14159266 & 45.00 & 30.00 & 0.57735027 \\
& $\mathrm{Sq}$ & 12.56637000 & 0.00 & 90.00 & 0.00000000 \\
Clamped & $\mathrm{Cr}$ & 12.56637000 & - & - & - \\
& $\mathrm{Tr}$ & 12.56637000 & 0.00 & 120.00 & 0.00000000 \\
\hline
\end{tabular}


RESTING
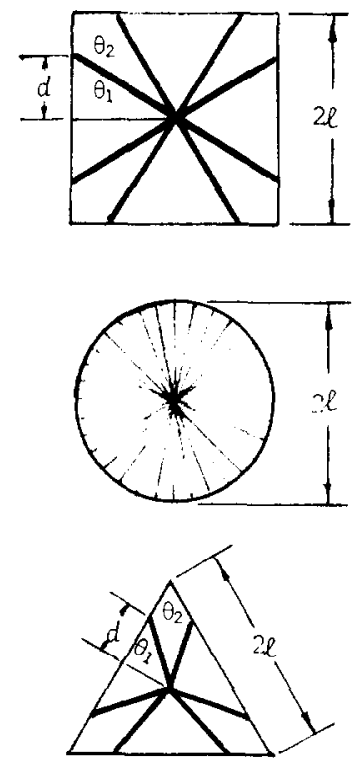

SIMPLY SUPPORTED
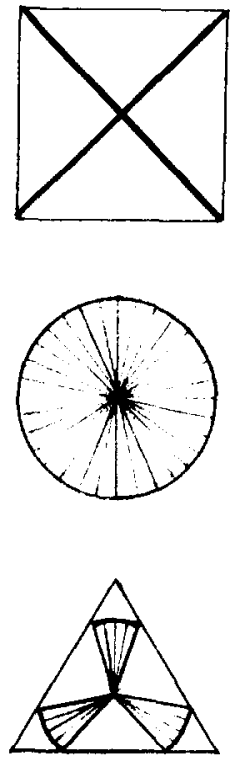

CLA:TPED
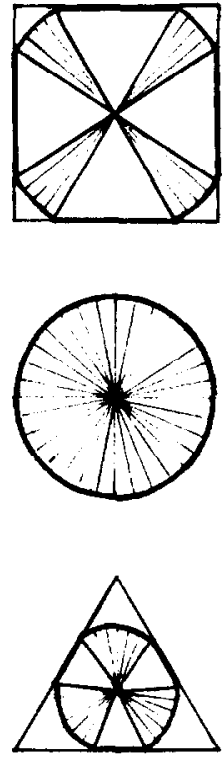

Fig. 1. Plate configurations and ridge line patterns.

$l$ and $d$ are defined in fig. 1 . The conical surfaces are shown as fans. The ridge lines are heavier than other lines. Only one independent parameter $\theta_{1}$ appears in minimization. Some solutions contained in [11] are reproduced here for verification.

\section{A cracked plate}

To present a more complex example, a simply supported square $(2 l \times 2 l)$ plate with a centered crack is considered in this section. The plate is under a uniform load and the crack has an arbitrary angle $\alpha$ and length $c$ as shown in fig. 2 . The effect of the crack on the load carrying capacity of the plate is studied by the method of limit analysis.

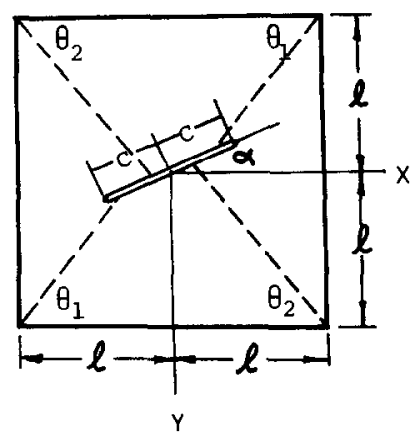

Fig. 2. Configuration and parameters of a cracked plate. 
The square plate suggests the ranges of $\alpha$ and $c$ such that

$$
0 \leq \alpha \leq 45^{\circ}, \quad 0 \leq c \leq l / \cos \alpha .
$$

The ridge lines shown in fig. 2 may or may not intersect the crack tips. The correct ridge lines will be determined by two angle parameters $\theta_{1}$ and $\theta_{2}$ which are optimized to obtain the limit load. The function $w(x, y)$ is assumed to be linear in each of the four subdomains separated by the ridge lines as shown. It is an easy exercise to calculate the integrals in (19) and construct the function

$$
q^{*}(w)=q^{*}\left(\theta_{1}, \theta_{2}, \alpha, c / l\right) .
$$

With two parameters $\alpha$ and $c / l$ assigned, $q^{*}(w)$ can be minimized with respect to $\theta_{1}$ and $\theta_{2}$. The dimensionless limit load of the cracked plate, $q_{L}^{*} l^{2} / 6 M_{0}$, is shown in fig. 3 for the entire ranges of $\alpha$ and $c / l$.

For small crack lengths, the orientation of the crack does not play a significant role in the limit load which decreases with increasing crack length. But the effect of the crack angle is quite pronounced at large crack lengths. It is also interesting to note that for any crack angle other than $45^{\circ}$, there is a range where crack length has no effect on the limit load. The limit load initially decreases with increasing crack length to a point then remains constant. The points separate the decreasing range from the constant range of the limit load are shown along the dashed curve.

The minimization procedure used in the $\theta_{1}-\theta_{2}$ space is the gradient descent method [9] which converges in 4 to 5 iterations for each set of $(\alpha, c / l)$. This fast convergence is achieved by the sequential computation such that a converged solution is used as the initial iterate for an adjacent pair $(\alpha, c / l)$.

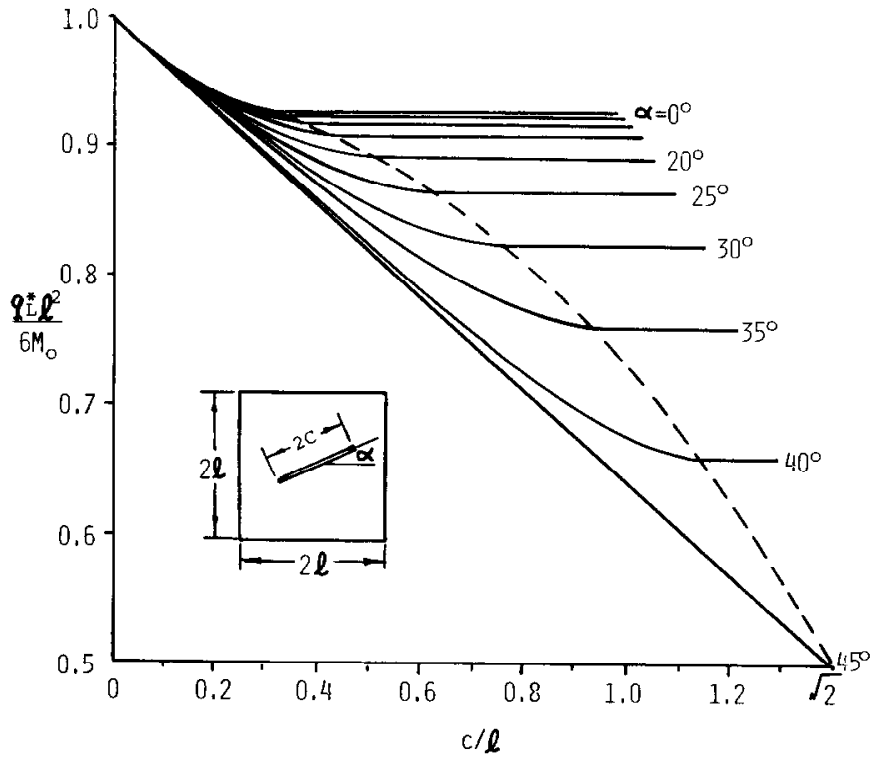

Fig. 3. Limit load of cracked plate with angle and length variations. 


\section{Final remarks}

Establishment of upper and lower bound solutions to limit analysis has been known for many years as indicated by a number of references listed. The list could have been much longer if a survey is intended. But a systematic search for the limit solution in a convex set using the developments of nonlinear programming methods is relatively recent. The computational methods certainly provide added power for solving more complex limit analysis problems. The minimization approach presented here computes the least upper bound from a given family of test functions (collapse modes). If the family contains all possible collapse modes, the minimum solution obtained is the limit solution. Otherwise, the minimum is an upper bound to the limit solution. Some solutions of the simple problems presented agree with the available known results. The other new solutions give physically correct quality and trend.

The solutions of the cracked plate problem point out a new phenomenon. For some configurations, the crack tip is not the most critical area of high stresses. This is contrary to the common belief developed from elastic analysis. The plastic analysis should give more realistic estimates on load capacity of ductile plates.

Limit analysis which studies the ultimate behavior rather than the elastoplastic response is a valuable aid to structural and machine designs. Its power can be better utilized when more convenient methods are made available. This paper and some similar developments [13, 14] offer a few effective methods. Methodology of limit analysis enjoyed much less development than that of elasticity.

\section{References}

[1] S.R. Daniels, Inelastic Steel Structures (University of Tennessee Press, Memphis, 1966).

[2] H.A. Balmer, J.St. Doltsinis and M. Konig, Elastoplastic and creep analysis with the ASKA program system, Comput. Meths. Appl. Mech. Engrg. 3 (1974) 87-104.

H.A. Balmer and J.St. Doltsinis, Extensions to the elastoplastic analysis with the ASKA program system, Comput. Meths. Appl. Mech. Engrg. 13 (1978) 363-401.

[3] T.G. Butler and D. Michel, NASTRAN; a summary of the functions and capacities of the NASA structural analysis computer system, Scientific and Technical Information Office, NASA SP-260 (1971).

[4] W. Prager and P.G. Hodge, Jr., Theory of Perfectly Plastic Solids (Wiley, New York, 1951).

[5] P.G. Hodge, Jr., Plastic Analysis of Structures (McGraw-Hill, New York, 1959).

[6] F.J. Plantema, Sandwich Construction, The Bending and Buckling of Sandwich Beams, Plates and Shells (Wiley, New York 1966).

[7] R.M. Jones, Mechanics of Composite Materials (McGraw-Hill, New York, 1975).

[8] S.P. Timoshenko and S. Woinowsky-Krieger, Theory of Plates and Shells (McGraw-Hill, New York 1959).

[9] D.G. Luenberger, Introduction to Linear and Nonlinear Programming (Addison-Wesley, Reading, MA, 1973).

[10] K. Washizu, Variational Methods in Elasticity and Plasticity (Pergamon Press, New York, 1975).

[11] E.H. Mansfield, Studies in collapse analysis of rigid-plastic plates with a square yield diagram, Proc. Roy. Soc. London Ser. A 241 (1957) 311-338.

[12] M.A. Save and C.E. Massonnet, Plastic Analysis and Design of Plates, Shclls and Discs (North-Holland, Amsterdam, 1972).

[13] W.H. Yang, On a class of optimization problems for framed structures, Comput. Meths. Appl. Mech. Engrg. 15 (1978) 85-98.

[14] W.H. Yang, A practical method for limit torsion problems, Comput. Meths. Appl. Mech. Engrg. 19 (1979) 151-158.

[15] D.C. Drucker, A definition of stable inelastic material, J. Appl. Mech. Trans. ASME 81 (E 101) (1959). 